\section{Determinantes dos gastos com diálises no Sistema Único de Saúde, Brasil, 2000 a 2004}

\author{
Determinants of expenditures on dialysis \\ in the Unified National Health System, \\ Brazil, 2000 to 2004
}

\begin{abstract}
The aim of this study was to compare total outpatient expenditures on hemodialysis and peritoneal dialysis from 2000 to 2004 in patients that began dialysis in 2000 under the Unified National Health System (SUS). A historical cohort was developed, consisting of patients that began dialysis in 2000, identified by probabilistic matching in the database of Authorizations for High-Complexity/High-Cost Procedures (APAC). A multiple linear regression model was used, including individual and clinical attributes and health services supply variables. The cohort included 10,899 patients, $88.5 \%$ of whom began hemodialysis and $11.5 \%$ peritoneal dialysis. The dialysis modality explains $12 \%$ of the variance in expenditures, and patients in peritoneal dialysis showed $20 \%$ higher mean annual expenditure. The differences in expenditures are explained according to the State of Brazil and health services supply level. Individual risk variables did not alter the model's explanatory power, while age and diabetes mellitus were significant. The study showed the importance of the National Health System's payment mechanism for explaining differences in expenditures on dialysis treatment in Brazil.
\end{abstract}

Renal Insufficiency; Dialysis; Health Expenditures; Health Economics

\author{
Mariangela Leal Cherchiglia 1 \\ Isabel Cristina Gomes 2 \\ Juliana Alvares 1 \\ Augusto Guerra Júnior 1 \\ Francisco de Assis Acúrcio 3 \\ Eli Iola Gurgel Andrade 1 \\ Alessandra Maciel Almeida 1 \\ Daniele Araújo Campo Szuster 1 \\ Mônica Viegas Andrade 4 \\ Odilon Vanni de Queiroz ${ }^{1}$
}

\section{Introdução}

A incidência da insuficiência renal crônica eleva-se no mundo, assim como os recursos alocados em seu tratamento. Ao final de 2004, aproximadamente, 1,8 milhão de pacientes estavam submetidos às terapias de substituição renal. Desses, 77\% encontravam-se em alguma forma de diálise, e $23 \%$ eram transplantados renais 1 . O impacto do tratamento de terapias de substituição renal no gasto total dos sistemas nacionais de saúde é relativamente elevado comparado às demais doenças. Independente do modelo assistencial adotado pelo sistema nacional de saúde, uma considerável quantidade de recursos financeiros é despendida com diálises - variando de $0,7 \%$ a $1,8 \%$ dos orçamentos em saúde - correspondendo à cobertura de um grupo relativamente pequeno da população ${ }^{2}$. Espera-se que os gastos com diálises, em todo mundo, ultrapassem os 200 bilhões de dólares americanos despendidos em 1990, alcançando mais de 1 trilhão no ano de 20103.

No Brasil, entre 2004 e 2007, houve um aumento do número de pacientes em terapias de substituição renal na ordem de $8,1 \%$ ao ano ${ }^{4}$. Os gastos com esses pacientes para o Sistema Único de Saúde (SUS), responsável por cerca de 95\% dos gastos com o tratamento renal, atingiram US\$ 400 mil no ano de 2004, sendo US\$ 300 mil utilizados para a cobertura das sessões dialíticas 5 . 
A despeito do elevado gasto com os procedimentos dialíticos, são escassos os estudos de âmbito nacional, de avaliação econômica, entre as modalidades de terapias de substituição renal no Brasil 6,7. Segundo Sancho \& Dain 8, é limitado o número de estudos empíricos que apresentam parâmetros, que permitam a elaboração de estudos de avaliação econômica sobre as terapias de substituição renal no Brasil. Tais restrições também são evidenciadas na literatura internacional 9,10,11. Just et al. 9 realizaram uma revisão sistemática das publicações em língua inglesa sobre as duas modalidades de diálise (hemodiálise e diálise peritoneal), no período de 1996 a 2006, e encontraram 62 artigos, dos quais, apenas 25 apresentavam análise econômica. De forma similar, Winkelmayer et al. 12 realizaram revisão da literatura de estudos, em língua inglesa, que apresentavam análise de custo-efetividade das terapias de substituição renal, no período de 1968 a 2000. Dos 764 artigos encontrados, somente 13 permitiram o cálculo da razão de custo-efetividade das modalidades de terapias de substituição renal. Dentre esses, apenas um era referente ao Brasil, o elaborado por Sesso et al. ${ }^{6}$. Os autores ressaltam a falta de consenso sobre a escolha da modalidade inicial e a dificuldade de se obter dados nacionais sobre gastos com tratamentos dialíticos, o que compromete a comparabilidade entre os estudos existentes 9,12.

O objetivo deste estudo é comparar os gastos ambulatoriais totais no SUS, no período de 2000 a 2004, dos pacientes incidentes que iniciaram diálise no ano de 2000, no Brasil, considerando as duas modalidades de terapias de substituição renal: hemodiálise e diálise peritoneal.

\section{Material e métodos}

\section{Desenho do estudo}

Trata-se de um estudo do tipo observacional, prospectivo e não concorrente, utilizando, como fonte de dados, a Base Nacional em Terapia Renal Substitutiva. Essa base foi desenvolvida por meio da técnica de relacionamento determinísticoprobabilístico, utilizando os bancos de dados da Autorização de Procedimentos de Alta Complexidade/Custo (APAC) do Sistema de Informações Ambulatoriais (SIA) do SUS e o Sistema de Informações sobre Mortalidade (SIM), com o objetivo de habilitar o seguimento dos pacientes na coorte 13,14 .

Utilizaram-se, também, as informações provenientes da Pesquisa Assistência Médico Sanitária do Instituto Brasileiro de Geografia e Estatística (AMS/IBGE) 15, realizada em 2003. A AMS investiga todos os estabelecimentos de saúde, públicos ou privados, constituindo-se em um censo da oferta de serviços de saúde no Brasil.

\section{População do estudo}

A população do estudo compreendeu todos os pacientes incidentes com registros de APAC/SIA, no período de janeiro a dezembro de 2000. Todos os pacientes foram acompanhados até a ocorrência do óbito ou até o final do estudo, em 31 de dezembro de 2004 (48 meses de seguimento). Foram considerados elegíveis os pacientes que apresentaram, pelo menos, três meses consecutivos de registros ao início do tratamento, dada a evidência de altas taxas de mortalidade nesse período e de possível ocorrência de registros de pacientes renais agudos 16,17. Foram excluídos os pacientes que receberam transplante renal durante o período, os que apresentaram cobrança de procedimento específico para HIV e os que não completaram quatro anos de acompanhamento, por perda de seguimento.

No ano de 2000, havia 17.422 pacientes incidentes. Foram excluídos 5.859 pacientes: 2.334 não apresentaram, pelo menos, três meses consecutivos de registros ao início do tratamento 2.278 receberam transplante renal durante o período de seguimento, 67 apresentaram alguma cobrança de procedimento específico para HIV, e 1.180 não completaram quatro anos de acompanhamento, por perda de seguimento.

Com relação às Unidades da Federação, foram excluídas as que apresentavam menos de vinte indivíduos por modalidade de tratamento, por não permitir estimar os modelos controlando por Unidade da Federação: Acre, Amapá, Maranhão, Mato Grosso do Sul, Mato Grosso, Roraima, Tocantins e Rondônia. No total, foram excluídas 664 observações. Ao final, nossa amostra foi composta por 10.899 pacientes incidentes. Ao comparar os pacientes elegíveis e não elegíveis para o estudo, observou-se que apresentavam características semelhantes segundo as variáveis analisadas. A modalidade de entrada foi definida como aquela na qual o paciente permaneceu por, pelo menos, três meses consecutivos ao início do tratamento.

\section{Variáveis}

A caracterização do perfil dos pacientes por modalidade de diálise foi realizada por meio de variáveis demográficas (sexo, idade, Unidade da Federação de residência no primeiro registro observado) e clínicas (diagnóstico ou não de diabetes mellitus como causa básica de insuficiência renal crônica). 
No modelo econométrico, foram incluídas as variáveis de oferta de cuidado renal relacionadas ao número de equipamentos de hemodiálise disponíveis no município de residência do paciente, no primeiro registro, e número de leitos per capita.

\section{Aferição de gastos}

A análise dos gastos adotou a perspectiva do financiador público e limitou-se aos gastos diretos da atenção à saúde. Para aferir os gastos por paciente, identificou-se um rol de 679 códigos de procedimentos distintos cobrados na APAC, que, após análise por painel de nefrologistas, resultou na seleção de 46 procedimentos, 47 medicamentos e dez exames relacionados às terapias de substituição renal. $\mathrm{O}$ gasto individual foi calculado somando-se os valores pagos por procedimentos, medicamentos e exames registrados a cada mês na APAC. Considerando que os procedimentos de diálise são ininterruptos, foi realizado um processo de imputação para as lacunas de registros mensais de cobrança verificadas entre o primeiro mês de observação e a ocorrência do óbito ou o término de acompanhamento do estudo. A técnica hot deck foi utilizada para imputação de dados, identificando conjuntos completos de gastos de indivíduos com características similares, por períodos de 12 meses, e, a partir desses, foram estimados os valores nas lacunas 18. Para os gastos com medicamentos e exames, não houve imputação de dados.

A partir desses dados, gerou-se uma tabela com as informações de valores mensais totais despendidos (em R\$) por paciente. O gasto médio individual anual foi calculado dividindo-se o gasto total aferido para cada paciente pelo número de meses de seu seguimento na coorte. O resultado obtido foi multiplicado por 12 . Os valores foram atualizados para dezembro de 2008 com base no Índice Nacional de Preços ao Consumidor Amplo (IPCA/IBGE; http://www. ibge.gov.br).

Devido à distribuição assimétrica dos gastos, optou-se por excluir os valores extremos (outliers) de gasto médio anual, adotando-se o método do escore padronizado ${ }^{19}$. O cálculo do escore padronizado considera o gasto médio anual de cada observação, subtraído da média dos gastos médios anuais e dividido pelo desviopadrão. Valores com escores acima de 3,3 foram considerados outliers e excluídos da análise. De acordo com a Desigualdade de Chebyschev, a probabilidade de o escore padronizado dos gastos assumir valores entre -3,3 e 3,3 é superior a $90 \%$, garantindo, assim, uma boa cobertura da amostra por esse critério. O ponto de corte obti- do foi o valor de $\mathrm{R} \$ 41.837,40$, que gerou a exclusão de 125 indivíduos (1\% dos participantes).

\section{Análise estatística}

Foi realizada análise descritiva, incluindo distribuições de freqüências, medidas de tendência central e de variabilidade para as características estudadas. O teste $\mathrm{z}$ foi utilizado para comparação de médias entre as modalidades de tratamento, considerando variância diferente entre as duas populações agrupadas (hemodiálise e diálise peritoneal). O nível de significância adotado foi $5 \%$.

Para avaliar os determinantes do gasto médio anual nos anos de 2000 a 2004, foi construído um modelo de regressão linear múltipla, incluindo, como covariáveis, os atributos individuais (idade, sexo, Unidade da Federação de residência do paciente), os fatores clínicos (presença de diabetes como causa da insuficiência renal) e variáveis de oferta associadas aos municípios de residência dos indivíduos no primeiro registro. A variável dependente é o logaritmo do gasto médio anual durante o período de 2000 a 2004. A especificação dos gastos na forma logarítmica é usualmente recomendada, dado que a distribuição de gastos se aproxima mais da distribuição log-normal. Além disso, tal especificação permite a interpretação dos resultados em termos de variação percentual dos gastos ao invés de valores absolutos. Foram estimados seis modelos.

O primeiro modelo inclui, como variável independente, apenas a modalidade de tratamento, e o segundo, as variáveis associadas ao risco individual (idade e sexo). A idade foi incluída como uma função polinomial de terceira ordem de forma a permitir maior flexibilidade na associação entre idade e gastos. No terceiro modelo, incluiu-se a variável que controla para presença do diagnóstico de diabetes. O quarto modelo inclui as dummies que controlam para Unidade da Federação de residência no primeiro registro de terapias de substituição renal. Essas dummies são proxies para a oferta de tratamento renal nos respectivos estados. O pressuposto é que a oferta de cuidado renal é relativamente homogênea, uma vez que a gestão do cuidado de alta complexidade é de responsabilidade estadual. O modelo 5 inclui as variáveis de oferta local, associadas ao município de residência do indivíduo no primeiro registro em terapias de substituição renal. Nesse caso, utilizou-se dummies tanto para os equipamentos de hemodiálise como para os leitos per capita, sendo a ausência de oferta a categoria de referência nos dois casos. No modelo 6 , foram introduzidas variáveis de interação entre a modalidade de tratamento e as variáveis de idade 
e sexo. A hipótese é que a relação entre gastos e modalidade pode ser diferente entre as idades ou entre os sexos.

A qualidade do ajuste foi verificada por meio do teste de Wald, do coeficiente de determinação e da análise de resíduos. Para todos os modelos, realizaram-se os testes de heterocedasticidade e, no caso de presença, os modelos foram reestimados considerando a matriz de correção da variância. Foram também realizados os testes de multicolinearidade entre as variáveis (teste VIF). Em todas as especificações, as variáveis não apresentaram problemas de correlação linear.

Os modelos foram estimados considerando duas bases de dados. Na primeira, todos os pacientes incidentes durante o ano de 2000, conforme já descrito acima. Na segunda base de dados, foram excluídos os pacientes que apresentaram a causa indeterminada como causa básica da insuficiência renal crônica. Esses pacientes totalizam 3.791 observações. A distribuição dos pacientes em ambas as bases de dados é bastante similar, de modo que são apresentados os resultados apenas para a base que exclui os indivíduos com causa indeterminada. Nesse caso, os pacientes apresentam-se mais homogêneos, permitindo comparar as diferenças de gastos entre as duas modalidades de tratamento. A análise estatística foi realizada utilizando-se o programa Stata 9 (Stata Corp., College Station, Estados Unidos).

Esta investigação integra o Projeto TRS - Avaliação Econômico-Epidemiológica das Terapias renais Substitutivas no Brasil - conduzido pelo Grupo de Pesquisa em Economia da Saúde da Universidade Federal de Minas Gerais (GPES/ UFMG), que visa conhecer a situação, o desenvolvimento e os resultados da política de assistência aos portadores de insuficiência renal crônica terminal no Brasil. O projeto de investigação foi aprovado pelo Comitê de Ética em Pesquisa da UFMG (parecer ETIC 397/2004).

\section{Resultados}

\section{Caracterização dos pacientes}

A coorte inicial foi constituída por 10.899 pacientes incidentes que iniciaram seu tratamento durante o ano de 2000 , dos quais, $88,5 \%$ entraram na modalidade hemodiálise, e $11,5 \%$, na modalidade diálise peritoneal. A maior proporção era do sexo masculino (56,1\%), com mais de 45 anos (72\%), sendo a idade média ao início de tratamento de 53,6 anos (DP $\pm 16,3$ anos). A maioria dos pacientes residia na Região Sudeste: $26,6 \%$ em São Paulo, $13 \%$ no Rio de Janeiro e $11,7 \%$ em Minas Gerais. Pacientes com diagnóstico de diabetes mellitus como causa de insuficiência renal à entrada em diálise representaram $20 \%$ (Tabela 1 ).

Considerando a modalidade inicial, observaram-se diferenças nas características demográficas e clínicas dos pacientes. Dos pacientes que iniciaram em hemodiálise, a maioria era do sexo masculino (56,9\%), e, entre os que iniciaram diálise peritoneal, observou-se distribuição homogênea entre os sexos. A idade média foi inferior para os que iniciaram em hemodiálise (53,3 anos; $\mathrm{DP} \pm 15,9$ ), quando comparados aos pacientes em diálise peritoneal $(55,4$ anos; $\mathrm{DP} \pm 19,3)$. O grupo etário que apresentou maior freqüência foi o de 45 a 64 anos, tanto para hemodiálise como para diálise peritoneal, mas observa-se maior proporção de pacientes acima de 65 anos iniciando em diálise peritoneal em relação à hemodiálise $(34,3 \%$ vs. $25,2 \%$; p < 0,05). Considerando as causas de doenças renais crônicas, observou-se que os pacientes em diálise peritoneal apresentaram maior proporção de diabetes mellitus (22\%) como causa primária de insuficiência renal crônica em comparação aos em hemodiálise.

A alocação inicial de pacientes entre as modalidades em relação à Unidade da Federação de residência revela grande concentração e disparidades. O Estado de São Paulo concentrava 34,9\% de todos os pacientes em diálise peritoneal, no país, naquele ano. Destaca-se que $70 \%$ dos pacientes, em ambas as modalidades, encontravam-se nas regiões Sudeste e Sul, e que as regiões Norte e Centro-Oeste apresentavam pacientes em tratamento dialítico em apenas dois estados. Em alguns estados como Alagoas, Amazonas, Bahia, Paraná e Distrito Federal, não se observou preponderância de uma modalidade sobre a outra, sugerindo condições semelhantes de oferta entre as duas modalidades.

\section{Caracterização dos gastos segundo modalidade de tratamento}

As Tabelas 2 e 3 apresentam as médias de gastos totais entre as modalidades de tratamento considerando os atributos individuais (idade, sexo e Unidade da Federação de residência) e presença de diabetes como causa da insuficiência renal crônica, bem como o coeficiente de variação.

Quando se analisam as diferenças entre os gastos segundo os atributos individuais, novamente observou-se que o gasto médio com diálise peritoneal é sempre superior ao de hemodiálise $(R \$ 26.810,00$ e $R \$ 33.870,00 ; p<0,005)$. A relação de gastos entre diálise peritoneal comparada à hemodiálise é de 1,26. Essa relação pouco varia quando são analisados os atributos individuais e clínicos, sendo as maiores variações para pacien- 
Tabela 1

Características dos pacientes que iniciaram terapia de substituição renal em 2000, segundo modalidade inicial de tratamento. Brasil, 2000 a 2004

\begin{tabular}{|c|c|c|c|c|c|c|c|}
\hline \multirow[t]{2}{*}{ Características } & \multicolumn{2}{|c|}{$\begin{array}{l}\text { Hemodiálise } \\
\begin{array}{c}(\mathrm{N}=9.651 ; \\
88,5 \%) *\end{array}\end{array}$} & \multicolumn{2}{|c|}{$\begin{array}{l}\text { Diálise peritoneal } \\
\qquad \begin{array}{c}(N=1.248 \\
11,5 \%)^{*}\end{array}\end{array}$} & \multicolumn{2}{|c|}{$\begin{array}{c}\text { Total } \\
(\mathrm{N}=10.899 ; \\
100,0 \%) \text { * }\end{array}$} & \multirow[t]{2}{*}{$\begin{array}{l}\text { Valor } \\
\text { de } p\end{array}$} \\
\hline & $\mathbf{n}$ & $\%$ & $\mathbf{n}$ & $\%$ & $\mathbf{n}$ & $\%$ & \\
\hline \multicolumn{8}{|l|}{ Sexo } \\
\hline Feminino & 4.155 & 43,1 & 633 & 50,7 & 4.788 & 43,9 & * \\
\hline Masculino & 5.496 & 56,9 & 615 & 49,3 & 6.111 & 56,1 & \\
\hline \multicolumn{8}{|c|}{ Idade à entrada em terapia de substituição renal ** } \\
\hline Média & \multicolumn{2}{|c|}{53,33} & \multicolumn{2}{|c|}{55,45} & \multicolumn{2}{|c|}{53,57} & * \\
\hline Desvio-padrão & \multicolumn{2}{|c|}{15,87} & \multicolumn{2}{|c|}{19,26} & \multicolumn{2}{|c|}{16,3} & \\
\hline \multicolumn{8}{|l|}{ Faixa etária (anos) ** } \\
\hline $0-19$ & 247 & 2,6 & 89 & 7,2 & 336 & 3,1 & * \\
\hline $20-44$ & 2.513 & 26,2 & 192 & 15,5 & 2.705 & 25,0 & \\
\hline $45-64$ & 4.419 & 46,1 & 531 & 43,0 & 4.950 & 45,7 & \\
\hline Acima de 65 & 2.413 & 25,2 & 424 & 34,3 & 2.837 & 26,2 & \\
\hline \multicolumn{8}{|c|}{ Unidade da Federação de residência } \\
\hline Alagoas & 240 & 2,5 & 30 & 2,4 & 270 & 2,5 & \\
\hline Amazonas & 118 & 1,2 & 10 & 0,8 & 128 & 1,2 & \\
\hline Bahia & 513 & 5,3 & 58 & 4,6 & 571 & 5,2 & \\
\hline Ceará & 344 & 3,6 & 10 & 0,8 & 354 & 3,2 & * \\
\hline Distrito Federal & 155 & 1,6 & 31 & 2,5 & 186 & 1,7 & \\
\hline Espírito Santo & 150 & 1,6 & 58 & 4,6 & 208 & 1,9 & * \\
\hline Goiás & 185 & 1,9 & 41 & 3,3 & 226 & 2,1 & * \\
\hline Minas Gerais & 1.151 & 11,9 & 122 & 9,8 & 1.273 & 11,7 & * \\
\hline Pará & 109 & 1,1 & 38 & 3,0 & 147 & 1,3 & * \\
\hline Paraíba & 302 & 3,1 & 48 & 3,8 & 350 & 3,2 & \\
\hline Pernambuco & 490 & 5,1 & 28 & 2,2 & 518 & 4,8 & * \\
\hline Piauí & 112 & 1,2 & 6 & 0,5 & 118 & 1,1 & * \\
\hline Paraná & 564 & 5,8 & 75 & 6,0 & 639 & 5,9 & \\
\hline Rio de Janeiro & 1.294 & 13,4 & 122 & 9,8 & 1.416 & 13,0 & * \\
\hline Rio Grande do Norte & 201 & 2,1 & 23 & 1,8 & 224 & 2,1 & * \\
\hline Rio Grande do Sul & 844 & 8,7 & 47 & 3,8 & 891 & 8,2 & * \\
\hline Santa Catarina & 285 & 3,0 & 25 & 2,0 & 310 & 2,8 & * \\
\hline Sergipe & 26 & 0,3 & 41 & 3,3 & 67 & 0,6 & * \\
\hline São Paulo & 2.568 & 26,6 & 435 & 34,9 & 3.003 & 27,6 & * \\
\hline Total & 9.651 & 100,0 & 1.248 & 100,0 & 10.899 & 100,0 & \\
\hline Causa de insuficiência renal & & & & & & & 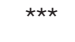 \\
\hline Diabetes mellitus & 1.903 & 19,7 & 275 & 22,0 & 2.178 & 20,0 & \\
\hline Não diabéticos & 7.748 & 80,3 & 973 & 78,0 & 8.721 & 80,0 & \\
\hline
\end{tabular}

Fonte: Base Nacional em Terapia Renal Substitutiva.

* Valor de $\mathrm{p}<0,05$.

** 71 registros não apresentavam data de nascimento

$\star \star \star$ Valor de $\mathrm{p}<0,10$; teste de qui-quadrado realizado para variáveis categóricas e t de Student para variáveis contínuas.

tes acima de 65 anos $(1,24)$, sexo feminino e com diagnóstico de diabetes $(1,27)$, e a menor para pacientes jovens $(1,23)$. Na análise dos gastos por estados federados, de residência do paciente, no- ta-se grande heterogeneidade dos valores entre estados e mesmo entre as modalidades. Observa-se que os gastos com diálise peritoneal são superiores aos de hemodiálise em todos os es- 
Tabela 2

Gastos médios anuais dos pacientes em terapia de substituição renal por modalidade, segundo as variáveis demográficas (faixa etária e sexo) e clínica (diagnóstico ou não de diabetes mellitus como causa básica de insuficiência renal crônica). Brasil, 2000 a 2004

\begin{tabular}{|c|c|c|c|c|}
\hline Variáveis & Hemodiálise & Diálise peritoneal & $\begin{array}{c}\text { Proporção diálise } \\
\text { peritoneal/ } \\
\text { hemodiálise }\end{array}$ & Valor de $p$ \\
\hline \multicolumn{5}{|l|}{ Modalidade } \\
\hline Média & $26.810,30$ & $33.870,50$ & 1,26 & * \\
\hline Erro-padrão & $3.936,46$ & $8.032,40$ & & \\
\hline Coeficiente de variação & 7,49 & 12,10 & & \\
\hline $\mathrm{n}$ & 9.651 & 1.248 & & \\
\hline \multicolumn{5}{|l|}{ Faixa etária (anos) } \\
\hline \multicolumn{5}{|l|}{$0-19$} \\
\hline Média & $27.592,63$ & $33.953,40$ & 1,23 & * \\
\hline Erro-padrão & $3.806,16$ & $7.122,42$ & & \\
\hline Coeficiente de variação & 7,04 & 10,70 & & \\
\hline $\mathrm{n}$ & 247 & 89 & & \\
\hline \multicolumn{5}{|l|}{$20-44$} \\
\hline Média & $26.863,40$ & $33.218,19$ & 1,24 & * \\
\hline Erro-padrão & $3.469,79$ & $7.826,58$ & & \\
\hline Coeficiente de variação & 6,59 & 12,02 & & \\
\hline $\mathrm{n}$ & 2.513 & 192 & & \\
\hline \multicolumn{5}{|l|}{$45-64$} \\
\hline Média & $26.928,73$ & $33.297,59$ & 1,24 & * \\
\hline Erro-padrão & $3.851,89$ & $7.905,25$ & & \\
\hline Coeficiente de variação & 7,30 & 12,11 & & \\
\hline $\mathrm{n}$ & 4.419 & 531 & & \\
\hline \multicolumn{5}{|l|}{ Acima de 65} \\
\hline Média & $26.478,41$ & $34.886,99$ & 1,32 & * \\
\hline Erro-padrão & $4.503,18$ & $8.401,23$ & & \\
\hline Coeficiente de variação & 8,68 & 12,29 & & \\
\hline $\mathrm{n}$ & 2.413 & 424 & & \\
\hline \multicolumn{5}{|l|}{ Sexo } \\
\hline \multicolumn{5}{|l|}{ Feminino } \\
\hline Média & $26.808,92$ & $34.099,17$ & 1,27 & * \\
\hline Erro-padrão & $4.035,62$ & $7.901,12$ & & \\
\hline Coeficiente de variação & 7,68 & 11,82 & & \\
\hline $\mathrm{n}$ & 4.155 & 633 & & \\
\hline \multicolumn{5}{|l|}{ Masculino } \\
\hline Média & $26.811,70$ & $33.635,14$ & 1,25 & * \\
\hline Erro-padrão & $3.860,18$ & $8.165,05$ & & \\
\hline Coeficiente de variação & 7,35 & 12,39 & & \\
\hline $\mathrm{n}$ & 5.496 & 615 & & \\
\hline \multicolumn{5}{|c|}{ Causa de insuficiência renal crônica } \\
\hline \multicolumn{5}{|l|}{ Diabéticos } \\
\hline Média & $27.269,95$ & $34.755,92$ & 1,27 & * \\
\hline Erro-padrão & $4.135,45$ & $7.959,03$ & & \\
\hline Coeficiente de variação & 7,74 & 11,68 & & \\
\hline $\mathrm{n}$ & 1.903 & 275 & & \\
\hline
\end{tabular}

(continua) 
Tabela 2 (continuação)

\begin{tabular}{lccc}
\hline Variáveis & Hemodiálise & Diálise peritoneal & $\begin{array}{c}\text { Proporção diálise } \\
\text { peritoneal/ } \\
\text { hemodiálise }\end{array}$ \\
\hline Não diabéticos & $26.697,66$ & $33.620,26$ & 1,26 \\
Média & $3.877,99$ & $8.039,40$ & \\
Erro-padrão & 7,41 & 12,20 & \\
Coeficiente de variação & 7.748 & 973 & \\
$n$ & & & \\
\hline
\end{tabular}

Fonte: Base Nacional em Terapia Renal Substitutiva.

Nota: despesas ambulatoriais e medicamentos de alto custo para os pacientes incidentes com registros no Sistema de Informações Ambulatoriais (SIA/SUS), no período de janeiro a dezembro de 2000 e acompanhados até dezembro de 2004; valores atualizados para dezembro de 2008 pelo Índice de Preços ao Consumidor Amplo do Instituto Brasileiro de Geografia e Estatística (IPCA/IBGE).

* Valor de $\mathrm{p}<0,05$; teste t de Student.

Tabela 3

Gastos médios anuais dos pacientes em terapia de substituição renal por modalidade, segundo a variável demográfica (Unidade da Federação de residência no primeiro registro observado). Brasil, 2000 a 2004.

\begin{tabular}{|c|c|c|c|c|}
\hline Unidade da Federação & Hemodiálise & Diálise peritoneal & $\begin{array}{c}\text { Proporção diálise } \\
\text { peritoneal/ } \\
\text { hemodiálise }\end{array}$ & Valor de $p$ \\
\hline \multicolumn{5}{|l|}{ Alagoas } \\
\hline Média & $28.723,52$ & $22.124,08$ & 0,77 & * \\
\hline Erro-padrão & $5.191,70$ & $10.677,80$ & & \\
\hline Coeficiente de variação & 9,22 & 24,62 & & \\
\hline$n$ & 240 & 30 & & \\
\hline \multicolumn{5}{|l|}{ Amazonas } \\
\hline Média & $30.391,76$ & $40.631,86$ & 1,34 & * \\
\hline Erro-padrão & $3.709,34$ & $2.304,35$ & & \\
\hline Coeficiente de variação & 6,23 & 2,89 & & \\
\hline$n$ & 118 & 10 & & \\
\hline \multicolumn{5}{|l|}{ Bahia } \\
\hline Média & $27.025,46$ & $32.756,13$ & 1,21 & * \\
\hline Erro-padrão & $3.763,24$ & $8.920,96$ & & \\
\hline Coeficiente de variação & 7,10 & 13,90 & & \\
\hline$n$ & 513 & 58 & & \\
\hline \multicolumn{5}{|l|}{ Ceará } \\
\hline Média & $28.861,37$ & $35.402,55$ & 1,23 & * \\
\hline Erro-padrão & $4.673,00$ & $7.915,88$ & & \\
\hline Coeficiente de variação & 8,26 & 11,41 & & \\
\hline $\mathrm{n}$ & 344 & 10 & & \\
\hline \multicolumn{5}{|l|}{ Distrito Federal } \\
\hline Média & $25.797,81$ & $28.439,56$ & 1,10 & * \\
\hline Erro-padrão & $2.469,06$ & $6.356,56$ & & \\
\hline Coeficiente de variação & 4,88 & 11,40 & & \\
\hline $\mathrm{n}$ & 155 & 31 & & \\
\hline
\end{tabular}

(continua) 
Tabela 3 (continuação)

\begin{tabular}{|c|c|c|c|c|}
\hline Unidade da Federação & Hemodiálise & Diálise peritoneal & $\begin{array}{c}\text { Proporção diálise } \\
\text { peritoneal/ } \\
\text { hemodiálise }\end{array}$ & Valor de $\mathrm{p}$ \\
\hline \multicolumn{5}{|l|}{ Espírito Santo } \\
\hline Média & $26.097,10$ & $33.016,34$ & 1,27 & * \\
\hline Erro-padrão & $3.224,23$ & $8.553,81$ & & \\
\hline Coeficiente de variação & 6,30 & 13,22 & & \\
\hline $\mathrm{n}$ & 150 & 58 & & \\
\hline \multicolumn{5}{|l|}{ Goiás } \\
\hline Média & $26.866,78$ & $27.187,26$ & 1,01 & 0.7837 \\
\hline Erro-padrão & $2.492,47$ & $7.333,28$ & & \\
\hline Coeficiente de variação & 4,73 & 13,76 & & \\
\hline $\mathrm{n}$ & 185 & 41 & & \\
\hline \multicolumn{5}{|l|}{ Minas Gerais } \\
\hline Média & $25.170,00$ & $30.503,96$ & 1,21 & * \\
\hline Erro-padrão & $3.463,68$ & $8.632,44$ & & \\
\hline Coeficiente de variação & 7,02 & 14,44 & & \\
\hline $\mathrm{n}$ & 1.151 & 122 & & \\
\hline \multicolumn{5}{|l|}{ Paraíba } \\
\hline Média & $22.941,79$ & $24.442,10$ & 1,07 & 0.2565 \\
\hline Erro-padrão & $4.569,18$ & $7.585,90$ & & \\
\hline Coeficiente de variação & 10,16 & 15,83 & & \\
\hline $\mathrm{n}$ & 109 & 38 & & \\
\hline \multicolumn{5}{|l|}{ Pará } \\
\hline Média & $26.566,88$ & $35.074,14$ & 1,32 & * \\
\hline Erro-padrão & $3.884,48$ & $6.396,00$ & & \\
\hline Coeficiente de variação & 7,46 & 9,30 & & \\
\hline $\mathrm{n}$ & 302 & 48 & & \\
\hline \multicolumn{5}{|l|}{ Pernambuco } \\
\hline Média & $27.568,78$ & $38.507,79$ & 1,40 & * \\
\hline Erro-padrão & $3.869,08$ & $4.550,88$ & & \\
\hline Coeficiente de variação & 7,16 & 6,03 & & \\
\hline $\mathrm{n}$ & 490 & 28 & & \\
\hline \multicolumn{5}{|l|}{ Piauí } \\
\hline Média & $27.506,84$ & $39.039,33$ & 1,42 & * \\
\hline Erro-padrão & $4.359,27$ & $2.218,15$ & & \\
\hline Coeficiente de variação & 8,09 & 2,90 & & \\
\hline $\mathrm{n}$ & 112 & 6 & & \\
\hline \multicolumn{5}{|l|}{ Paraná } \\
\hline Média & $26.493,65$ & $35.574,38$ & 1,34 & * \\
\hline Erro-padrão & $3.546,74$ & $5.244,79$ & & \\
\hline Coeficiente de variação & 6,83 & 7,52 & & \\
\hline$n$ & 564 & 75 & & \\
\hline \multicolumn{5}{|l|}{ Rio de Janeiro } \\
\hline Média & $27.319,84$ & $36.827,51$ & 1,35 & * \\
\hline Erro-padrão & $3.962,92$ & $6.212,80$ & & \\
\hline Coeficiente de variação & 7,40 & 8,61 & & \\
\hline$n$ & 1.294 & 122 & & \\
\hline
\end{tabular}

(continua) 
Tabela 3 (continuação)

\begin{tabular}{|c|c|c|c|c|}
\hline Unidade da Federação & Hemodiálise & Diálise peritoneal & $\begin{array}{c}\text { Proporção diálise } \\
\text { peritoneal/ } \\
\text { hemodiálise }\end{array}$ & Valor de $p$ \\
\hline \multicolumn{5}{|l|}{ Rio Grande do Norte } \\
\hline Média & $25.987,07$ & $33.236,40$ & 1,28 & * \\
\hline Erro-padrão & $4.329,49$ & $5.453,18$ & & \\
\hline Coeficiente de variação & 8,50 & 8,37 & & \\
\hline $\mathrm{n}$ & 201 & 23 & & \\
\hline \multicolumn{5}{|l|}{ Rio Grande do Sul } \\
\hline Média & $24.798,46$ & $37.789,85$ & 1,52 & * \\
\hline Erro-padrão & $3.450,57$ & $5.095,20$ & & \\
\hline Coeficiente de variação & 7,10 & 6,88 & & \\
\hline $\mathrm{n}$ & 844 & 47 & & \\
\hline \multicolumn{5}{|l|}{ Santa Catarina } \\
\hline Média & $27.243,63$ & $35.039,15$ & 1,29 & * \\
\hline Erro-padrão & $3.801,01$ & $7.897,46$ & & \\
\hline Coeficiente de variação & 7,12 & 11,50 & & \\
\hline$n$ & 285 & 25 & & \\
\hline \multicolumn{5}{|l|}{ Sergipe } \\
\hline Média & $27.246,36$ & $29.983,65$ & 1,10 & 0,0668 \\
\hline Erro-padrão & $3.325,90$ & $8.396,60$ & & \\
\hline Coeficiente de variação & 6,23 & 14,29 & & \\
\hline $\mathrm{n}$ & 26 & 41 & & \\
\hline \multicolumn{5}{|l|}{ São Paulo } \\
\hline Média & $27.487,51$ & $35.820,72$ & 1,30 & * \\
\hline Erro-padrão & $3.594,76$ & $6.880,66$ & & \\
\hline Coeficiente de variação & 6,67 & 9,80 & & \\
\hline $\mathrm{n}$ & 2.568 & 435 & & \\
\hline
\end{tabular}

Fonte: Base Nacional em Terapia Renal Substitutiva.

Nota: despesas ambulatoriais e medicamentos de alto custo para os pacientes incidentes com registros no Sistema de Informações Ambulatoriais (SIA/SUS), no período de janeiro a dezembro de 2000 e acompanhados até dezembro de 2004; valores atualizados para dezembro de 2008 pelo Índice de Preços ao Consumidor Amplo do Instituto Brasileiro de Geografia e Estatística (IPCA/IBGE).

* Valor de $\mathrm{p}<0,05$; teste t de Student.

tados, exceto em Alagoas, alcançando os valores mais altos no Estado do Amazonas (R\$40.600,00 para diálise peritoneal e $\mathrm{R} \$ 30.300,00$ para hemodiálise). Quando os gastos são agregados por região, observa-se que a Região Sul apresenta o maior gasto médio em diálise peritoneal, e a Região Nordeste, em hemodiálise. O menor gasto médio para diálise peritoneal é encontrado na Região Centro-Oeste e, para hemodiálise, na Região Sul. Os menores valores de diálise peritoneal e hemodiálise foram encontrados em Alagoas e na Paraíba, respectivamente. Entretanto, a maior variação na relação de gastos entre diálise peritoneal e hemodiálise é observada no Rio Grande do Sul $(1,52)$. Os estados de Goiás, Sergipe, Paraíba e Distrito Federal apresentaram os menores valo- res na relação de gastos entre diálise peritoneal e hemodiálise $(1,01$ a 1,10).

Avaliando-se o coeficiente de variação, observa-se que o da diálise peritoneal é sempre maior do que o da hemodiálise para os atributos modalidade, faixa etária, sexo e causa de insuficiência renal crônica. Em relação às Unidades da Federação, o coeficiente de variação para diálise peritoneal é inferior ao de hemodiálise para os estados do Amazonas, Pernambuco, Piauí, Rio Grande do Norte e Rio Grande do Sul.

\section{Análise da determinação dos gastos}

As Tabelas 4 e 5 reportam os resultados encontrados para os seis modelos estimados. Os re- 
Determinação do gasto médio anual da diálise pelos modelos 1, 2 e 3. Brasil, 2000 a 2004.

\begin{tabular}{|c|c|c|c|c|c|c|c|c|c|c|c|c|}
\hline \multirow[t]{3}{*}{ Variáveis } & \multicolumn{4}{|c|}{ Modelo 1} & \multicolumn{4}{|c|}{ Modelo 2} & \multicolumn{4}{|c|}{ Modelo 3} \\
\hline & \multirow[t]{2}{*}{$\beta$} & \multicolumn{2}{|c|}{ IC95\% } & \multirow{2}{*}{$\begin{array}{l}\text { Valor } \\
\text { de } p\end{array}$} & \multirow[t]{2}{*}{$\beta$} & \multicolumn{2}{|c|}{ IC95\% } & \multirow{2}{*}{$\begin{array}{l}\text { Valor } \\
\text { de } p\end{array}$} & \multirow[t]{2}{*}{$\beta$} & \multicolumn{2}{|c|}{ IC95\% } & \multirow{2}{*}{$\begin{array}{l}\text { Valor } \\
\text { de } p\end{array}$} \\
\hline & & LI & LS & & & LI & LS & & & LI & LS & \\
\hline Modalidade & $-0,208$ & $-0,218$ & $-0,197$ & * & $-0,209$ & $-0,221$ & $-0,195$ & * & $-0,208$ & $-0,229$ & $-0,186$ & * \\
\hline Sexo & & & & & 0,000 & $-0,007$ & 0,009 & & 0,000 & $-0,007$ & 0,010 & \\
\hline Idade (anos) & & & & & $-0,003$ & $-0,008$ & $-0,001$ & $\star \star \star$ & $-0,003$ & $-0,008$ & 0,000 & $\star \star$ \\
\hline Idade (quadrática) & & & & & 0,000 & 0,000 & 0,000 & $\star \star \star$ & 0,000 & 0,000 & 0,000 & \\
\hline Idade (cúbica) & & & & & 0,000 & 0,000 & 0,000 & $\star \star \star *$ & 0,000 & 0,000 & 0,000 & $\star \star *$ \\
\hline Diabetes & & & & & & & & & 0,024 & 0,004 & 0,023 & * \\
\hline Constante & 10,3 & & & & 10,300 & & & * & 10,300 & & & * \\
\hline R2 \# & 0,122 & & & & 0,124 & & & & 0,126 & & & \\
\hline
\end{tabular}

IC95\%: intervalo de 95\% de confiança.

Fonte: Base Nacional em Terapia Renal Substitutiva.

Nota: categorias de referência - modalidade de hemodiálise; sexo feminino e não diabético.

* Valor de $\mathrm{p}<0,001$;

** Valor de $\mathrm{p}<0,05$;

$\star \star \star$ Valor de $\mathrm{p}<0,01$;

\# Constante e R2: coeficientes de determinação que indicam o percentual da variabilidade da resposta que foi explicado pelo modelo.

sultados são bastante estáveis, e os coeficientes apresentam pequena alteração com a inclusão de novas variáveis. O grau de ajuste do modelo completo, cerca de $20 \%$, evidencia relativo poder de explicação das covariáveis. A análise do coeficiente referente ao tipo de modalidade revela que - estar em diálise peritoneal aumenta em cerca de $20 \%$ os gastos médios individuais anuais. Esse coeficiente praticamente não se altera entre os modelos 1 a 3 . No modelo 6 , o coeficiente se altera porque foi incluída a variável de interação entre a modalidade e idade. Em relação à presença de diagnóstico de diabetes mellitus, os resultados encontrados indicam que, embora ter diagnóstico de diabetes como causa primaria incremente os gastos, essa variação é relativamente pequena, cerca de $2 \%$. Esse resultado é estatisticamente significativo e muito robusto na magnitude. A análise dos modelos 4 e 5 revela que as variáveis de estado de residência no início da diálise e de oferta de serviços de saúde são bastante relevantes para explicar os gastos com terapias de substituição renal no Brasil, uma vez que o poder de explicação do modelo aumenta em cerca de $50 \%$, passando de $12 \%$ para quase $20 \%$. Observase estabilidade na variação dos gastos em relação aos estados, poucos casos (Distrito Federal, Paraíba e Sergipe) apresentam uma variação igual ou maior do que $10 \%$. Chama atenção o Estado da Paraíba que apresenta um gasto 25\% menor que o de São Paulo.

\section{Discussão}

Os resultados deste estudo demonstram que os pacientes que iniciaram o tratamento dialítico em diálise peritoneal apresentam um gasto médio anual cerca de $20 \%$ maior do que os que iniciaram em hemodiálise, sendo que a modalidade de diálise é responsável por $12 \%$ da variância dos gastos entre os pacientes. A inclusão das variáveis de risco individual praticamente não altera o poder de explicação do modelo. Entre os atributos de risco, apresentam diferenças estatisticamente significativas a idade e a presença de diabetes mellitus. Esses resultados reforçam a importância do sistema de pagamento das terapias renais no SUS para explicar as diferenças de gastos entre os pacientes, uma vez que esse sistema não discrimina as características individuais nem o perfil de risco. A existência dessa diferença foi também detectada em outros estudos de âmbito nacional 5,6.

Na maioria dos países desenvolvidos, a diálise peritoneal é menos custosa do que a hemodiálise, numa relação que varia entre 1,22 a 1,52 10,11,20, contrastando com os dados observados para a maioria dos países em desenvolvimento, no qual a modalidade de diálise peritoneal é a mais onerosa 9 . Nesse estudo, o valor da relação entre diálise peritoneal e hemodiálise é de 1,26. Segundo Just et al. ${ }^{9}$, os custos da hemodiálise são influenciados pelos custos fixos como estrutura 
Tabela 5

Determinação do gasto médio anual da diálise pelos modelos 4, 5 e 6. Brasil, 2000 a 2004.

\begin{tabular}{|c|c|c|c|c|c|c|c|c|c|c|c|c|}
\hline \multirow[t]{3}{*}{ Variáveis } & \multicolumn{4}{|c|}{ Modelo 4} & \multicolumn{4}{|c|}{ Modelo 5} & \multicolumn{4}{|c|}{ Modelo 6} \\
\hline & \multirow[t]{2}{*}{$\boldsymbol{\beta}$} & \multicolumn{2}{|c|}{ IC95\% } & \multirow{2}{*}{$\begin{array}{l}\text { Valor } \\
\text { de } p\end{array}$} & \multirow[t]{2}{*}{$\beta$} & \multicolumn{2}{|c|}{ IC95\% } & \multirow{2}{*}{$\begin{array}{l}\text { Valor } \\
\text { de } p\end{array}$} & \multirow[t]{2}{*}{$\beta$} & \multicolumn{2}{|c|}{ IC95\% } & \multirow{2}{*}{$\begin{array}{l}\text { Valo } \\
\text { de } p\end{array}$} \\
\hline & & LI & LS & & & LI & LS & & & LI & LS & \\
\hline Sexo & $-0,001$ & $-0,009$ & 0,007 & & $-0,002$ & $-0,009$ & 0,006 & & $-0,002$ & $-0,009$ & 0,006 & \\
\hline Modalidade & $-0,214$ & $-0,229$ & $-0,203$ & * & $-0,214$ & $-0,237$ & $-0,196$ & * & $-0,159$ & $-0,179$ & $-0,059$ & * \\
\hline Idade (anos) & $-0,003$ & $-0,008$ & $-0,001$ & $\star \star$ & $-0,003$ & $-0,009$ & $-0,001$ & $\star \star$ & $-0,004$ & $-0,009$ & $-0,001$ & $\star \star$ \\
\hline Idade (quadrática) & 0,000 & 0,000 & 0,000 & $\star \star$ & 0,000 & 0,000 & 0,000 & ** & 0,000 & 0,000 & 0,000 & 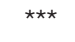 \\
\hline Idade (cúbica) & 0,000 & 0,000 & 0,000 & $\star \star$ & 0,000 & 0,000 & 0,000 & $\star \star \star$ & 0,000 & 0,000 & 0,000 & 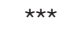 \\
\hline Diabetes & 0,021 & 0,009 & 0,026 & * & 0,021 & 0,007 & 0,025 & * & 0,020 & 0,007 & 0,025 & * \\
\hline \multicolumn{13}{|l|}{ Unidade da Federação } \\
\hline Alagoas & $-0,035$ & $-0,034$ & 0,013 & $\star \star \star$ & $-0,033$ & $-0,047$ & 0,034 & & $-0,034$ & $-0,047$ & 0,034 & \\
\hline Amazonas & 0,101 & 0,074 & 0,136 & * & 0,073 & 0,055 & 0,114 & * & 0,074 & 0,055 & 0,115 & * \\
\hline Bahia & $-0,027$ & $-0,022$ & 0,017 & * & $-0,030$ & $-0,021$ & 0,014 & * & $-0,030$ & $-0,021$ & 0,014 & * \\
\hline Ceará & 0,040 & 0,022 & 0,064 & * & 0,040 & 0,021 & 0,063 & * & 0,040 & 0,022 & 0,063 & * \\
\hline Distrito Federal & $-0,085$ & $-0,171$ & $-0,066$ & * & $-0,114$ & $-0,190$ & $-0,090$ & * & $-0,113$ & $-0,178$ & $-0,086$ & * \\
\hline Espírito Santo & 0,000 & $-0,120$ & $-0,043$ & & 0,000 & $-0,110$ & $-0,020$ & & 0,000 & $-0,109$ & $-0,020$ & \\
\hline Goiás & $-0,055$ & $-0,111$ & $-0,054$ & * & $-0,044$ & $-0,112$ & $-0,043$ & 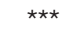 & $-0,043$ & $-0,111$ & $-0,043$ & 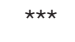 \\
\hline Minas Gerais & $-0,100$ & $-0,106$ & $-0,079$ & * & $-0,092$ & $-0,095$ & $-0,067$ & * & $-0,092$ & $-0,095$ & $-0,067$ & * \\
\hline Paraíba & $-0,252$ & $-0,266$ & $-0,187$ & * & $-0,258$ & $-0,297$ & $-0,166$ & * & $-0,256$ & $-0,295$ & $-0,164$ & * \\
\hline Pará & $-0,032$ & $-0,068$ & $-0,021$ & * & $-0,026$ & $-0,057$ & $-0,005$ & 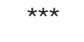 & $-0,026$ & $-0,057$ & $-0,005$ & 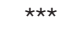 \\
\hline Pernambuco & 0,003 & $-0,016$ & 0,017 & & 0,010 & $-0,007$ & 0,023 & & 0,010 & $-0,007$ & 0,024 & \\
\hline Piauí & 0,002 & $-0,044$ & 0,036 & & 0,016 & $-0,028$ & 0,052 & & 0,016 & $-0,030$ & 0,049 & \\
\hline Paraná & $-0,031$ & $-0,055$ & $-0,022$ & * & $-0,027$ & $-0,045$ & $-0,014$ & * & $-0,027$ & $-0,045$ & $-0,014$ & * \\
\hline Rio Grande do Norte & $-0,062$ & $-0,079$ & $-0,026$ & * & $-0,054$ & $-0,074$ & $-0,018$ & * & $-0,054$ & $-0,073$ & $-0,018$ & * \\
\hline Rio Grande do Sul & $-0,094$ & $-0,113$ & $-0,080$ & * & $-0,086$ & $-0,101$ & $-0,071$ & * & $-0,085$ & $-0,100$ & $-0,069$ & * \\
\hline Santa Catarina & $-0,012$ & $-0,026$ & 0,023 & & 0,001 & $-0,012$ & 0,036 & & 0,001 & $-0,012$ & 0,036 & \\
\hline Sergipe & $-0,108$ & $-0,163$ & $-0,078$ & * & $-0,111$ & $-0,197$ & $-0,058$ & * & $-0,106$ & $-0,189$ & $-0,050$ & $\star \star \star$ \\
\hline \multicolumn{13}{|l|}{ Número de equipamentos de } \\
\hline \\
\hline $1-20$ & & & & & $-0,013$ & $-0,037$ & $-0,008$ & $\star \star$ & $-0,013$ & $-0,037$ & $-0,008$ & $\star \star$ \\
\hline $20-50$ & & & & & 0,000 & $-0,010$ & 0,014 & & 0,000 & $-0,010$ & 0,014 & \\
\hline $50-70$ & & & & & $-0,012$ & $-0,044$ & 0,007 & & $-0,012$ & $-0,044$ & 0,007 & \\
\hline $70-100$ & & & & & 0,034 & 0,005 & 0,049 & * & 0,034 & 0,005 & 0,048 & * \\
\hline$>100$ & & & & & 0,017 & 0,013 & 0,034 & * & 0,017 & 0,013 & 0,034 & * \\
\hline \multicolumn{13}{|l|}{ Número de leitos/habitantes } \\
\hline $0-1$ & & & & & 0,000 & & & & 0,000 & & & \\
\hline $1-3$ & & & & & 0,016 & $-0,001$ & 0,028 & 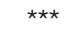 & 0,017 & $-0,001$ & 0,028 & 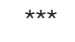 \\
\hline $3-5$ & & & & & 0,016 & $-0,006$ & 0,025 & ** & 0,016 & $-0,006$ & 0,025 & $\star \star$ \\
\hline$>5$ & & & & & 0,005 & $-0,020$ & 0,017 & & 0,006 & $-0,020$ & 0,017 & \\
\hline Interação idade vs. modalidade & & & & & & & & & $-0,001$ & $-0,003$ & $-0,001$ & ** \\
\hline Constante & 10,400 & & & * & 10,400 & & & * & 10,300 & & & * \\
\hline $\mathrm{R} 2$ \# & 0,195 & & & & 0,199 & & & & 0,200 & & & \\
\hline
\end{tabular}

IC95\%: intervalo de 95\% de confiança.

Fonte: Base Nacional em Terapia Renal Substitutiva.

Nota: categorias de referência - modalidade de hemodiálise; sexo feminino; Estado de São Paulo; não diabético, 0 equipamentos de hemodiálise por habitantes; 0 leitos por habitantes.

* Valor de $\mathrm{p}<0,001$;

** Valor de $\mathrm{p}<0,05$;

$\star \star \star$ Valor de $\mathrm{p}<0,01$;

\# Constante e R2: coeficientes de determinação que indicam o percentual da variabilidade da resposta que foi explicado pelo modelo. 
física, máquinas de diálises e tratamento da água e gastos com pessoal. Os custos com a diálise peritoneal derivam primariamente de custos variáveis, tais como soluções dialíticas e cateteres. Ao requerer menos tecnologia do que a hemodiálise, a diálise peritoneal poderia ser especialmente útil nos países em desenvolvimento. No entanto, nesses países, o custo com recursos humanos é mais baixo vis-à-vis aos custos de importação de equipamentos e solutos. Entre os países em desenvolvimento, o México e a África do Sul conseguiram superar a barreira do alto custo da importação de soluções para diálise peritoneal, tendo sido capazes de oferecer essa modalidade por um custo menor 21 .

Como mencionado, um importante resultado desse trabalho é a homogeneidade observada no gasto médio individual anual do tratamento dialítico, independente das características de risco. Uma das razões que podem explicar esse padrão de gastos no Brasil é a forma de financiamento da terapia renal no sistema de saúde brasileiro. O Ministério da Saúde remunera, por procedimentos, os serviços de diálise, estabelecendo valores fixos e padronizados para todo o território nacional, não considerando as diferenças regionais e individuais. O pagamento aos serviços de diálise é realizado por meio do subsistema de informação da APAC do SIA/SUS. Esse sistema é específico para autorização, cobrança e disponibilização de informações gerenciais de procedimentos de alta complexidade/custos (que incluem as terapias de substituição renal) e para o fornecimento de medicamentos excepcionais 22. Historicamente, o sistema de saúde remunerava a diálise peritoneal com valores mais altos do que a hemodiálise, como incentivo à disseminação dessa forma de cuidado. Entretanto, como o pagamento dos insumos utilizados para diálise peritoneal é feito diretamente às empresas fornecedoras, gera-se um incentivo financeiro negativo para os serviços de diálises e nefrologistas ao utilizarem essa modalidade de terapia. O ganho financeiro com essa modalidade se limita ao pagamento do treinamento inicial e de uma consulta mensal para acompanhamento, podendo gerar desinteresse dos médicos em prescrevê-la e dos serviços em provê-las 23. Segundo De Vecchi et al. 2, a escolha da modalidade é influenciada pelo valor pago para o procedimento e por outras diferenças inerentes aos custos, como a estrutura de lucro de uma terapia e pelos incentivos aos provedores para ofertá-la. Esses fatores são importantes e devem ser considerados quando se busca incentivar determinada modalidade de tratamento, alinhando os interesses dos diversos agentes econômicos do sistema de assistência no cuidado ao portador de doença renal crônica terminal 24 .
Vale mencionar que o Ministério da Saúde repassa, para estados e municípios, no caso das terapias de substituição renal, um valor global fixo segundo série histórica. O valor para diálise peritoneal, estipulado na tabela de procedimentos do SUS, é maior do que aquele pago para a hemodiálise, levando os gestores estaduais e municipais a optar pelo procedimento menos oneroso aos orçamentos próprios, colocando maiores entraves à liberação da diálise peritoneal 25 .

A análise do coeficiente de variação revela maior variabilidade para os gastos em diálise peritoneal comparados aos de hemodiálise. Em parte, esse comportamento pode estar associado às diferenças observadas nos tamanhos das amostras entre as duas terapias. A diferença de variabilidade é praticamente constante quando comparamos os gastos considerando os atributos individuais. Esse padrão se diferencia entre as duas terapias principalmente quando os estados da federação são considerados, sugerindo variabilidade de preços regionais de outros componentes do cuidado que estão sujeitos a diferenciais de preços em função dos mercados locais.

Além da modalidade, os resultados do modelo revelam que os diferenciais nos gastos são explicados pelo estado da federação, bem como pelo nível de oferta de serviços de saúde no município de residência do paciente, no início do tratamento dialítico. As variáveis - estado de residência e oferta de serviços de saúde - foram bastante relevantes para explicar os gastos com as diálises no Brasil. Como mencionado, a responsabilidade da gestão dos procedimentos de alto custo, como as diálises, no SUS, é, em sua maioria, atribuída aos estados mais do que aos municípios. A suposição é que os pacientes que vivem num mesmo estado têm acesso similar às diálises. A importância do estado de residência no início do tratamento para explicar os gastos também pode estar associada com a iniqüidade no acesso aos serviços de saúde nas diferentes regiões do Brasil 26,27. Em 2003, os residentes nas regiões Sudeste e Sul tiveram maior acesso aos serviços de saúde do que os residentes nas outras regiões, apesar da melhoria nesse item, com exceção da Região Norte 27 . Destaca-se que $63,5 \%$ dos nefrologistas, no Brasil, estão alocados em somente quatro estados 28 . Os estados da Região Nordeste apresentam, em sua maioria, um gasto menor do que São Paulo, com destaque para o Estado da Paraíba que gasta $25 \%$ a menos do que São Paulo. O impacto nos gastos decorrente das variáveis de oferta pode ser explicado pela correlação entre oferta, serviços de saúde e maior consumo dos mesmos, especialmente a disponibilidade de exames de alto custo e medicamentos de uso excepcional, como a eritropo- 
etina que é utilizada pelos pacientes em diálise para tratamento da anemia 26,27,29. Para Sancho \& Dain ${ }^{8}$, na avaliação econômica das diálises, dois fatores teriam maior impacto na alocação de recursos: a utilização de eritropoetina e a admissão hospitalar. Harris 30 relata que, na Austrália, os gastos com diálise, apesar de serem relativamente homogêneos, são afetados pela região de residência do pacientes, especialmente as regiões que apresentam baixa densidade populacional, e pela oferta de serviços de saúde. Outros autores observaram as mesmas tendências para os Estados Unidos e Canadá ${ }^{31,32}$. As razões para essa associação são complexas e são influenciadas pelas preferências de pacientes e provedores de serviços de saúde e principalmente pela política de saúde vigente.

Por último, os valores dos gastos observados nesse estudo segundo os atributos individuais para as modalidades hemodiálise e diálise peritoneal apresentam baixa variabilidade, sugerindo a existência de uma padronização de valores, determinada, principalmente, pela fixação dos preços pelo SUS como já discutido. A observação da interação entre a modalidade e idade revelou que as diferenças de gastos entre as modalidades ocorrem de forma diferenciada ao longo das idades. Em relação à presença de diagnóstico de diabetes mellitus, os resultados encontrados indicam que, embora esse diagnóstico incremente os gastos, a variação é relativamente pequena, cerca de $2 \%$. A literatura indica que as diferenças individuais - estado clínico, idade, presença de comorbidade e internações - são fatores capazes de influenciar os gastos do cuidado integral ao indivíduo 9,10,31. Entretanto, neste estudo, a não incorporação de gastos com internação constitui-se em uma limitação, uma vez que impactam diretamente nos gastos individuais.

\section{Conclusão}

Este estudo apresenta os gastos reais do sistema de saúde brasileiro para a manutenção anual de pacientes em procedimentos dialíticos, comparando as modalidades hemodiálise e diálise peritoneal. Os maiores gastos foram observados para pacientes tratados na modalidade diálise peritoneal. A forma de pagamento para tratamento dialítico estabelecida pelo SUS - por procedimentos e com valores fixos no território nacional - pode estar associada com a predominância de pacientes em hemodiálise e a homogeneidade observada nos gastos médios individuais.

Em parte, isso se deve também à escolha da modalidade, fato que não ocorre em um vácuo econômico. Pode-se dizer que existe um com- plexo de interesses, bem como um fluxo direto e indireto de recursos financeiros entre os participantes da cadeia do cuidado dialítico - pacientes, médicos, provedores de serviços de diálise e produtores e distribuidores de equipamentos e suprimentos, entre outros. Quando os incentivos entre os participantes não estão alinhados, podese observar, como neste estudo, que, apesar de a diálise peritoneal constituir um custo maior para o Ministério da Saúde, ela representa, na perspectiva dos provedores de serviços de diálise, um baixo rendimento ou ganho marginal.

Como os resultados clínicos das duas modalidades são similares, e o Ministério da Saúde vem tentando aprimorar a assistência aos pacientes submetidos às terapias de substituição renal, faz-se necessário considerar e implementar mudanças na forma de pagamento dos procedimentos dialíticos, no sentido de prevenir incentivos negativos e incorporar as diferenças regionais e de riscos individuais na implementação de um cuidado integral ao paciente portador de doença renal.

Este trabalho, ao comparar os gastos ambulatoriais do SUS entre as duas modalidades de diálise a partir da Base Nacional em Terapia Renal Substitutiva, é um passo para o desenvolvimento de parâmetros que possibilitem estudos de avaliação econômica nessas terapias no Brasil. A agenda futura indica a realização de análises econômicas completas, como a análise de custo-efetividade e custo/utilidade das alternativas de tratamento da insuficiência renal crônica, subsidiando a formulação de políticas públicas sobre diálise no Brasil. 


\section{Resumo}

O objetivo deste estudo é comparar os gastos ambulatoriais totais entre hemodiálise e diálise peritoneal, de 2000 a 2004, dos pacientes que iniciaram diálise, em 2000, no Sistema Único de Saúde (SUS). Foi desenvolvida coorte histórica de pacientes que iniciaram diálise em 2000, identificados por pareamento probabilístico na base de dados de Autorização de Procedimentos de Alta Complexidade/Custo (APAC). Utilizou-se modelo de regressão linear múltipla incluindo atributos individuais, clínicos e variáveis de oferta de serviços de saúde. A coorte foi constituída por 10.899 pacientes, 88,5\% iniciaram em hemodiálise, e 11,5\%, em diálise peritoneal. A modalidade explica $12 \%$ da variância dos gastos, os pacientes em diálise peritoneal apresentam um gasto médio anual $20 \%$ maior. Os diferenciais nos gastos são explicados pelo estado da federação e nível de oferta de serviços de saúde. As variáveis de risco individual não alteram o poder de explicação do modelo, sendo significativos a idade e a presença de diabetes mellitus. Constata-se a importância do sistema de pagamento do SUS para explicar as diferenças de gastos do tratamento dialítico no Brasil.

Insuficiência Renal; Diálise; Gastos em Saúde; Economia da Saúde

\section{Referências}

1. Grassmann A, Moeller S, Brown G. ESRD patients in 2004: global overview of patient numbers, treatment modalities and associated trends. Nephrol Dial Transplant 2005; 20:2587-93.

2. De Vecchi AF, Dratwa M, Wiedemann ME. Health care systems and end-stage renal disease (ESRD) therapies - an international review: costs and reimbusement/funding of ESRD therapies. Nephrol Dial Transplant 1999; 14 Suppl 6:31-41.

3. Lysaght MJ. Maintenance dialysis population dynamics: current trends and long-term implications. J Am Soc Nephrol 2002; 13 Suppl 1:S37-40.

4. Sesso R, Silva RB, Kowalski SC, Manfredi SR, Canziani ME, Draibe SA, et al. Dialysis care, cardiovascular disease, and costs in end-stage renal disease in Brazil. Int J Technol Assess Health Care 2007; 23:126-30.

\section{Colaboradores}

M. L. Cherchiglia participou da concepção, desenho, análise e interpretação dos dados, revisão crítica do conteúdo e aprovação da versão final do manuscrito. I. C. Gomes colaborou na análise e interpretação dos dados e aprovação da versão final do manuscrito. J. Alvares, A. M. Almeida e D. A. C. Szuster contribuíram na análise e interpretação dos dados, revisão crítica do conteúdo e aprovação da versão final do manuscrito. A. Guerra Júnior, F. A. Acúrcio e E. I. G. Andrade participaram da concepção e planejamento, revisão crítica do conteúdo e aprovação da versão final do manuscrito. M. V. Andrade colaborou na concepção, desenho, análise e interpretação dos dados, revisão crítica do conteúdo e aprovação da versão final do manuscrito. O. V. Queiroz contribuiu na concepção e planejamento e aprovação da versão final do manuscrito.

\section{Agradecimentos}

Os autores agradecem os integrantes do Grupo de Pesquisa em Economia da Saúde da Universidade Federal de Minas Gerais por sua valorosa contribuição desde o desenho metodológico à revisão final. Ao apoio financeiro do Fundo Nacional de Saúde/Ministério da Saúde (Convênio no. 4864/2005), Conselho Nacional de Desenvolvimento Científico e Tecnológico (CNPq; processo 409729/2006-0) e Fundação de Amparo à Pesquisa do Estado de Minas Gerais (FAPEMIG, processo EDT 3284/06)
5. Oliveira MB, Romão Jr. JE, Zatz R. End-stage renal disease in Brazil: epidemiology, prevention and treatment. Kidney Int Suppl 2005; (97):S82-6.

6. Sesso RCC, Stabile C, Draibe SA, Ajzen H, Ramos OL. Custo-efetividade do tratamento da insuficiência renal crônica terminal no Brasil. J Bras Nefrol 1987; 9:45-54.

7. Sesso R, Lopes AA, Thomé FS, Bevilacqua JL, Romão Jr. JE, Lugon J. Resultados do censo de diálise da SBN, 2008. J Bras Nefrol 2008; 30:233-8.

8. Sancho LG, Dain S. Análise de custo-efetividade em relação às terapias renais substitutivas: como pensar estudos em relação a essas intervenções no Brasil? Cad Saúde Pública 2008; 24:1279-90. 
9. Just PM, Riella MC, Tschosik EA, Noe LL, Bhattacharyya SK, de Charro F. Economic evaluations of dialysis treatment modalities. Health Policy 2008; 86:163-80.

10. Baboolal K, McEwan P, Sondhi S, Spiewanowski P, Wechowski J, Wilson K. The cost of renal dialysis in a UK setting: a multicentre study. Nephrol Dial Transplant 2008; 23:1982-9.

11. Peeters P, Rublee D, Just PM, Joseph A. Analysis and interpretation of cost data in dialysis: review of western European literature. Health Policy 2000; 54:209-27.

12. Winkelmayer WC, Weinstein MC, Mittleman MA, Glynn RJ, Pliskin JS. Health economic evaluations: the special case of end-stage renal disease treatment. Med Decis Making 2002; 22:417-30.

13. Cherchiglia ML, Guerra Júnior AA, Andrade EIG, Machado CJ, Acúrcio FA, Meira Júnior W, et al. A construção da base de dados nacional em terapia renal substitutiva (TRS) centrada no indivíduo: aplicação do método de linkage determinístico-probabilístico. Rev Bras Estud Popul 2007; 24:163-7.

14. Queiroz OV, Guerra Júnior AA, Machado CJ, Andrade EIG, Meira Junior W, Acúrcio FA, et al. A construção da Base Nacional em Terapia Renal Substitutiva (TRS) centrada no indivíduo: relacionamento entre registros de óbitos do subsistema de Autorização de Procedimentos de Alta Complexidade (APAC) e Sistema de Informações de Mortalidade (SIM). Epidemiol Serv Saúde 2009; 18:107-20.

15. Instituto Brasileiro de Geografia e Estatística. Pesquisa de assistência médico sanitária [CD-ROM]. Rio de Janeiro: Instituto Brasileiro de Geografia e Estatística; 2002.

16. Agodoa LY, Eggers PW. Renal replacement therapy in the United States: data from the United States Renal Data System. Am J Kidney Dis 1995; 25: 119-33.

17. Metcalfe W, Khan IH, Prescott GJ, Simpson K, MacLeod AM. Can we improve early mortality in patients receiving renal replacement therapy? Kidney Int 2000; 57:2539-45.

18. Boscarioli C. Pré-processamento de dados para descoberta de conhecimento em banco de dados: uma visão geral. In: Anais do III CONGED - Congresso de Tecnologias para Gestão de Dados e Metadados do Cone Sul. Guarapuava: Unicentro Editora; 2005. p. 101-20.

19. Meyer PL. Probabilidade: aplicações à estatística. Rio de Janeiro: Livros Técnicos e Científicos; 1984.

20. Shih YC, Guo A, Just PM, Mujais S. Impact of initial dialysis modality and modality switches on Medicare expenditures of end-stage renal disease patients. Kidney Int 2005; 68:319-29.
21. Barsoum R. Overview: end-stage renal disease in the developing world. Artif Organs 2002; 26: 737-46.

22. Secretaria de Atenção à Saúde, Ministério da Saúde. Sistema de Informações Ambulatoriais do SUS (SIA/SUS): manual de orientações técnicas. Brasília: Ministério da Saúde; 2006.

23. Secretaria de Atenção à Saúde, Ministério da Saúde. Portaria SAS nº. 393 de 20 de setembro de 2001. Faculta ao prestador de serviços de Terapia Renal Substitutiva - TRS proceder à cessão de seus créditos, a favor de suas empresas fornecedoras, referentes aos procedimentos conjunto de troca para DPA/DPAC. http://dtr2001.saude.gov.br/sas/ PORTARIAS/Port2001/PT-393.htm (acessado em 23/Jan/2007)

24. Neil N, Steven G, Wong L, Inglese G, Bhattacharyya SK, Gehr T, et al. The financial implications for Medicare of greater use of peritoneal dialysis. Clin Ther 2009; 31:880-8.

25. Ugá MA, Piola SF, Porto SM, Vianna, SM. Descentralização e alocação de recursos no âmbito do Sistema Único de Saúde (SUS). Ciênc Saúde Coletiva 2003; 8:417-37.

26. Paniz VMV, Fassa AG, Facchini LA, Bertoldi AD, Piccini RX, Tomasi E, et al. Acesso a medicamentos de uso contínuo em adultos e idosos nas regiões Sul e Nordeste do Brasil. Cad Saúde Pública 2008; 24:267-80

27. Travassos C, Oliveira EXG, Viacava F. Desigualdades geográficas e sociais no acesso aos serviços de saúde no Brasil: 1998 e 2003. Ciênc Saúde Coletiva 2006; 11:975-86.

28. Machado MH, Pinto LF, Castro A, Cenzi J. Perfil dos nefrologistas no Brasil. J Bras Nefrol 2000; 22(3 Suppl 2):10-6.

29. Costa JSD, Olinto MTA, Gigante DP, Menezes AMB, Macedo S, Fuchs SC. Cobertura do exame físico de mama: estudo de base populacional em Pelotas, RS. Rev Bras Epidemiol 2003; 6:39-48.

30. Harris A. The organization and funding of the treatment of end-stage renal disease in Australia. Int J Health Care Finance Econ 2007; 7:113-32.

31. Collins AJ, Chen SC, Gilbertson DT, Foley RN. CKD surveillance using administrative data: impact on the health care system. Am J Kidney Dis 2009; 53(3 Suppl 3):S27-36.

32. Prakash S, Austin PC, Oliver MJ, Garg AX, Blake PG Hux JE. Regional effects of satellite haemodialysis units on renal replacement therapy in non-urban Ontario, Canada. Nephrol Dial Transplant 2007; 22:2297-303.

Recebido em 03/Set/2009

Versão final reapresentada em 21/Dez/2009

Aprovado em 29/Jun/2010 